\title{
Relação entre gênero e sintomas depressivos e ansiosos em tabagistas
}

\author{
Relationship between gender and depressive and anxiety symptoms in smokers
}

\author{
Maria da Graça Tanori de Castro ${ }^{1}$, Margareth da Silva Oliveira ${ }^{2}$, Renata Brasil Araujo ${ }^{3}$, Rosemeri \\ Siqueira Pedroso ${ }^{4}$
}

${ }^{1}$ Mestre em Psicologia Clínica, Pontifícia Universidade Católica do Rio Grande do Sul (PUCRS), Porto Alegre, RS. Psiquiatra. Professora, Pós-Graduação Lato Sensu em Psiquiatria, Instituto Abuchaim, Porto Alegre, RS. ${ }^{2}$ Doutora. Professora, Pós-Graduação em Psicologia, PUCRS. ${ }^{3}$ Doutora. Supervisora de estágio em Dependência Química, Centro de Estudos José de Barros Falcão, Fundação Faculdade Federal de Ciências Médicas de Porto Alegre (FFFCMPA), Porto Alegre, RS. ${ }^{4}$ Psicóloga. Mestre em Psicologia Clínica.

\section{Resumo}

Introdução: O tabagismo apresenta, freqüentemente, comorbidade com ansiedade e depressão, patologias que são mais freqüentes entre as mulheres.

Objetivo: O objetivo deste estudo foi avaliar a associação entre o grau de dependência de nicotina e os sintomas de ansiedade e depressão em homens e mulheres tabagistas.

Método: Foi realizado um estudo transversal, com uma amostra por conveniência de 475 tabagistas da população geral. Os instrumentos utilizados foram: o Questionário de Tolerância de Fagerström, o Inventário de Depressão de Beck, o Inventário de Ansiedade de Beck e uma Ficha com dados sociodemográficos

Resultados: Nas mulheres, os sintomas de ansiedade $(F=9,691 ; p<0,01)$ e depressão $(F=16,23 ; p<0,01)$ apresentaram associação com a gravidade da dependência da nicotina. Entre os homens, apenas os sintomas de ansiedade $(F=7,219 ; \mathrm{p}=0,001)$ estiveram associados com o grau de dependência de nicotina.

Conclusão: As tabagistas mais graves têm mais sintomas de ansiedade e depressão, enquanto os tabagistas mais graves apresentam mais ansiedade. Estes achados apontam para a importância de avaliar esses tipos de sintomas ao serem tratados tabagistas no Brasil.

Descritores: Tabagismo, ansiedade, depressão.

\begin{abstract}
Introduction: Smoking often presents comorbid anxiety and depression, disorders that are more frequent in women.

Objective: The aim of this study was to evaluate the association between severity of nicotine dependence and anxiety and depression symptoms in both male and female smokers.

Method: A cross-sectional study was performed using a convenience sample of 475 smokers from the general population. The instruments used were Fagerström Test for Nicotine Dependence, Beck Depression Inventory, Beck Anxiety Inventory, and a social and demographic record sheet.

Results: In women, anxiety symptoms $(\mathrm{F}=9.691 ; \mathrm{p}<0.01)$ and depression $(\mathrm{F}=16.23 ; \mathrm{p}<0.01)$ were associated with severity of nicotine dependence. In men, only anxiety symptoms $(\mathrm{F}=7.219 ; \mathrm{p}=0.001)$ were associated with severity of nicotine dependence.

Conclusion: Women with severe nicotine dependence have more anxiety and depression symptoms, while severe male smokers only showed more anxiety. The results demonstrated the importance of evaluating these symptoms in the treatment of smokers in Brazil.

Keywords: Smoking, anxiety, depression.
\end{abstract}

\section{Introdução}

O tabagismo - que neste estudo pode ser entendido como dependência de nicotina - é um problema de saúde pública mundial que está associado a altas taxas de morbimortalidade. Embora sua prevalência esteja diminuindo nos países desenvolvidos, o mesmo não é observado nos países em desenvolvimento, mesmo com o 
aumento das campanhas educativas e do conhecimento dos riscos relacionados ao uso de tabaco, os quais estão bem definidos desde $1964^{1,2}$. No Brasil, um país considerado em desenvolvimento, o tabagismo tem uma prevalência de $10,1 \%$, segundo os dados do II Levantamento Domiciliar Sobre o Uso de Drogas Psicotrópicas realizado em 20053. Este segundo levantamento, realizado apenas 4 anos após o primeiro ${ }^{4}$, demonstrou um aumento de $1,1 \%$ na taxa de dependência do tabaco na população brasileira, o que, em termos de impacto em saúde pública, é bastante preocupante. No que diz respeito ao tabagismo feminino, verifica-se que houve uma redução maior no tabagismo entre os homens do que entre as mulheres ${ }^{5}$; outro estudo latino-americano aponta um maior consumo de tabaco entre as mulheres do que entre os homens ${ }^{6}$, sendo necessária maior atenção das políticas públicas para a população feminina de tabagistas ${ }^{5}$.

Segundo a Associação Psiquiátrica Americana ${ }^{7}$, cerca de $80 \%$ dos tabagistas gostaria de deixar de fumar, $35 \%$ param a cada ano, mas uma taxa inferior a $5 \%$ alcança esse objetivo sem auxílio externo.

Entre os vários fatores que dificultam a manutenção da abstinência da nicotina está o alto índice de comorbidade entre a dependência dessa substância e outras doenças psiquiátricas ${ }^{8-10}$. Observa-se que a tendência observada em algumas populações de diminuição da prevalência do tabagismo não é demonstrada entre os indivíduos que apresentam algum transtorno psiquiátrico. As altas taxas de morte prematura apresentadas por esta clientela, que são cinco vezes maior do que na população em geral, são atribuíveis às inúmeras doenças associadas à dependência de nicotina ${ }^{11}$.

As comorbidades psiquiátricas são, portanto, fatores importantes de serem considerados na avaliação de tabagistas. Schmitz et al. ${ }^{12}$, avaliando uma amostra de 3.293 tabagistas, encontraram que mais da metade dos sujeitos apresentava outro diagnóstico psiquiátrico. Grant et al. ${ }^{9}$ também encontraram forte associação entre tabagismo e outras patologias, centrando seu estudo em diagnósticos de eixo $I^{7}$.

Em relação à ocorrência conjunta de mais de um diagnóstico psiquiátrico entre os dependentes de tabaco, o abuso de outras substâncias, a depressão maior e os transtornos de ansiedade são as associações comórbidas mais prevalentes ${ }^{11}$.

Vários autores pesquisaram a associação entre o tabagismo e os transtornos de ansiedade ${ }^{12-15}$, o mesmo ocorrendo com relação à depressão ${ }^{15-18}$; no entanto, os mesmos não realizaram a avaliação da associação dos sintomas de ansiedade ou depressão com a gravidade da dependência da nicotina nos diferentes gêneros.

Podemos observar que os transtornos de ansiedade e a depressão, patologias freqüentemente associadas ao tabagismo, são mais prevalentes entre as mulheres ${ }^{19}$. Issa et al..$^{20}$ observaram que mulheres tabagistas apresentam quase o dobro de ansiedade e depressão que os homens tabagistas: $67 \%$ contra $38 \%$, respectivamente.

Considerando-se, então, que os transtornos de ansiedade e os de humor são comumente encontrados em associação com a dependência de tabaco e que há uma relação destes fatores com o gênero, este estudo teve por objetivo avaliar a associação entre o grau de dependência de nicotina e os sintomas de ansiedade e depressão em homens e mulheres tabagistas.

\section{Método}

Este estudo teve um delineamento transversal. A amostra, por conveniência, foi composta por 475 tabagistas da população geral, sendo $36,6 \%$ dos sujeitos do sexo masculino $(n=174)$ e $63,4 \%$ do sexo feminino $(n=301)$. A média de idade foi de 37,72 anos (desvio padrão, $\mathrm{DP}=$ $11,71 ; 18-65)$.

Os indivíduos foram recrutados pelo método snowball (bola de neve): tabagistas que trabalhavam em um hospital do Sistema Único de Saúde (SUS) indicavam outros tabagistas que iam, sucessivamente, indicando outros até que fosse completada a amostra. Os indivíduos indicados eram convidados por telefone pelos autores principais deste estudo, sendo-lhes explicado o objetivo do estudo e o fato de sua participação ser voluntária. Aqueles que concordavam em participar marcavam uma entrevista individual com um dos auxiliares de pesquisa (estudantes de psicologia treinados) na casa do sujeito ou outro local combinado.

O treinamento da equipe ocorreu previamente ao início da pesquisa, sendo feitas observações de aplicações realizadas por uma das coordenadoras do projeto até que as dúvidas dos auxiliares de pesquisa fossem sanadas.

Foram excluídos os sujeitos que apresentavam patologias clínicas tabaco-relacionadas ou que apresentavam sintomas psicóticos ou déficits cognitivos que alterassem o desempenho nos testes. A exclusão de indivíduos com patologias clínicas ocorreu por este ser um fator que poderia estar associado com sintomas de depressão e ansiedade, e, portanto, constituir um fator de confusão.

Os instrumentos utilizados foram: 1) ficha com dados sociodemográficos e informações gerais sobre saúde e história de uso do tabaco; 2) Questionário de Tolerância de Fagerström ${ }^{21,22}$, para avaliar a gravidade da dependência de nicotina; 3) Inventário de Depressão de Beck (BDI), para avaliar os sintomas depressivos, validado no Brasil por Cunha ${ }^{23}$, que definiu seus pontos de corte em: depressão mínima (de 0 a 11 pontos), leve (de 12 a 19 pontos), moderada (de 20 a 35 pontos) e grave (de 36 a 63 pontos); 4) Inventário de Ansiedade de Beck (BAI), que mede a intensidade dos sintomas de ansiedade e também foi validado no Brasil ${ }^{23}$. Os pontos de corte para a população brasileira são: ansiedade mínima (de 0 a 10 pontos), leve 
(de 11 a 19 pontos), moderada (de 20 a 30 pontos) e grave (de 31 a 63 pontos) $)^{23}$.

\section{Procedimentos}

Os instrumentos foram aplicados individualmente pela equipe treinada, iniciando pela ficha de dados sociodemográficos e dados referentes ao perfil de consumo do tabaco, de outras substâncias psicoativas e história médica. Aos sujeitos que preenchiam os critérios de inclusão foram aplicados os seguintes instrumentos: Escala de Fagerström, BDI e o BAI.

\section{Análise dos dados}

O banco de dados foi estruturado no Statistical Package for Social Sciences (SPSS), versão 12.0. A análise exploratória dos dados constou de testes estatísticos descritivos e de freqüências. $\mathrm{Na}$ análise inferencial, foram utilizados o teste qui-quadrado, o teste $t$ de Student para amostras independentes e a análise de variância (ANOVA) com o teste Tukey. O nível de significância utilizado como parâmetro foi o de 5\%.

\section{Aspectos éticos}

Esta pesquisa foi aprovada pelo Comitê de Ética em Pesquisa da Pontifícia Universidade Católica do Rio Grande do Sul (PUCRS). Os participantes foram informados dos procedimentos desta pesquisa, e os dados foram coletados após a assinatura do Termo de Consentimento Livre e Esclarecido.

\section{Resultados}

Os dados sociodemográficos e acerca do consumo de tabaco e sua comparação entre homens e mulheres encontram-se demonstrados na Tabela 1.

A gravidade da dependência de nicotina apresentou, de acordo com o coeficiente de correlação linear de Pearson, uma correlação de baixa intensidade com os sintomas de ansiedade avaliados pelo BDI $(r=0,260 ; p<$ $0,01)$ e com os sintomas de depressão avaliados pelo BAI $(\mathrm{r}=0,267 ; \mathrm{p}<0,01)$.

As mulheres apresentaram mais sintomas de depressão do que os homens, segundo o teste $t$ de Student para amostras independentes $(t=-3,336 ; \mathrm{p}<0,01)$. A média dos sintomas depressivos das mulheres foi de 11,41 (DP = $9,81$ ), e a dos homens, de 8,45 ( $\mathrm{DP}=7,74)$. No que diz respeito aos sintomas de ansiedade, também houve diferença entre os sexos $(t=-3,420 ; \mathrm{p}=0,001)$, sendo a média destes sintomas nas mulheres de 12,61 ( $\mathrm{DP}=11,37)$, e nos homens de 8,96 (DP = 10,13).

As médias dos sintomas de ansiedade e depressão, conforme os graus de dependência de nicotina nos homens e mulheres, encontram-se demonstrados, segundo a ANOVA, na Tabela 2.

As médias de pontos do BAI e BDI nos diferentes níveis de dependência de nicotina nos homens e mulheres

Tabela 1 - Dados sociodemográficos e referentes ao consumo de tabaco entre homens e mulheres

\begin{tabular}{lccc}
\hline Variáveis & Homens & Mulheres & p \\
\hline Idade & $38,63(11,79)^{*}$ & $37,19(11,64)^{*}$ & $0,200^{\dagger}$ \\
Anos de estudo & $11,27(2,81)^{*}$ & $11,74(3,11)^{*}$ & $0,095^{\dagger}$ \\
Idade de início do uso do tabaco & $16,58(3,88)^{*}$ & $16,88(3,66)^{*}$ & $0,486^{\dagger}$ \\
Tempo de uso do tabaco & $22,03(12,62)^{*}$ & $20,35(11,72)^{*}$ & $0,150^{\dagger}$ \\
Cigarros/dia & $18,43(10,92)^{*}$ & $17,13(11,10)^{*}$ & $0,217^{\dagger}$ \\
Fagerström total & $4,36(2,67)^{*}$ & $4,12(2,71)^{*}$ & $0,341^{\dagger}$ \\
Grau de dependência do tabaco & & & \\
$\quad$ Dependência leve & $80(46 \%)^{\ddagger}$ & $166(55,3 \%)^{\ddagger}$ & \\
$\quad$ Dependência moderada & $71(40,8 \%)^{\ddagger}$ & $94(31,3 \%)^{\ddagger}$ & $0,096^{\S}$ \\
$\quad$ Dependência alta & $23(13,2 \%)^{\ddagger}$ & $40(13,3 \%)^{\ddagger}$ & \\
Estado civil & & & \\
Solteiro & $72(41,9 \%)^{\ddagger}$ & $151(50,2 \%)^{\ddagger}$ & \\
Casado & $71(41,3 \%)^{\ddagger}$ & $96(31,9 \%)^{\ddagger}$ & $0,076^{\S}$ \\
Viúvo & $13(7,6 \%)^{\ddagger}$ & $34(11,3 \%)^{\ddagger}$ & \\
Separado & $16(9,3 \%)^{\ddagger}$ & $20(6,6 \%)^{\ddagger}$ & \\
\hline
\end{tabular}

* Média (desvio padrão).

$\uparrow$ Teste $t$ de Student para amostras independentes.

n $(\%)$.

$\S$ Teste qui-quadrado. 
apresentadas na Tabela 1 podem ser, para uma melhor visualização, observadas na Figura 1.

\section{Discussão}

Pode-se observar que não houve diferença significativa entre homens e mulheres quanto aos dados sociodemográficos e relacionados ao consumo de tabaco, o que permite que se comparem os dois grupos quanto aos aspectos relacionados aos sintomas de ansiedade e de depressão.

Neste estudo, o gênero não esteve associado à gravidade do tabagismo, assemelhando-se a outras pesquisas onde os autores também não encontraram esta diferença usando o Questionário de Tolerância de Fagerström ${ }^{14,24}$.
Em relação à quantidade de cigarros consumida, também não foi encontrada diferença entre os homens e as mulheres, ao contrário do estudo de Brady \& Randall ${ }^{25}$, no qual foi observado que as mulheres fumam menos do que os homens, embora os níveis de nicotina sejam similares, provavelmente devido ao metabolismo desta substância ser mais lento nas mulheres.

Um dado que chamou a atenção foi o fato de a ansiedade estar associada à gravidade da dependência de nicotina tanto para os homens como para as mulheres e da depressão só estar associada à gravidade nas mulheres, dado que contradiz os resultados do estudo de Alati et al. ${ }^{26}$. De acordo com Alati et al. ${ }^{26}$, os homens tabagistas apresentaram maiores índices de ansiedade do que os nãotabagistas; no entanto, não houve diferença quanto a estes índices nos diferentes graus da dependência de nicotina.

Tabela 2 - Comparação das médias pela análise de variância dos sintomas de ansiedade e depressão de acordo com o grau de dependência do tabaco e o gênero, média (desvio padrão)

\begin{tabular}{lccccc}
\hline Variáveis & Leve & Moderado & Grave & F & p \\
\hline Masculino & & & & & \\
$\quad$ Sintomas depressivos & $7,66(6,94)$ & $8,46(7,45)$ & $11,18(10,47)$ & 1,787 & 0,171 \\
$\quad$ Sintomas de ansiedade & $5,83(6,87)^{\mathrm{a}}$ & $11,65(11,93)^{\mathrm{b}}$ & $11,50(10,83)^{\mathrm{b}}$ & 7,219 & 0,001 \\
& & & & \\
Feminino & & & & \\
$\quad$ Sintomas depressivos & $8,96(7,76)^{\mathrm{a}}$ & $12,77(10,14)^{\mathrm{b}}$ & $18,03(12,58)^{\mathrm{c}}$ & 16,23 & $<0,01$ \\
$\quad$ Sintomas de ansiedade & $10,38(10,12)^{\mathrm{a}}$ & $13,82(12,25)^{\mathrm{b}}$ & $18,69(11,51)^{\mathrm{b}}$ & 9,691 & $<0,01$ \\
\hline
\end{tabular}

Teste Tukey: letras iguais indicam ausência de diferença significativa.

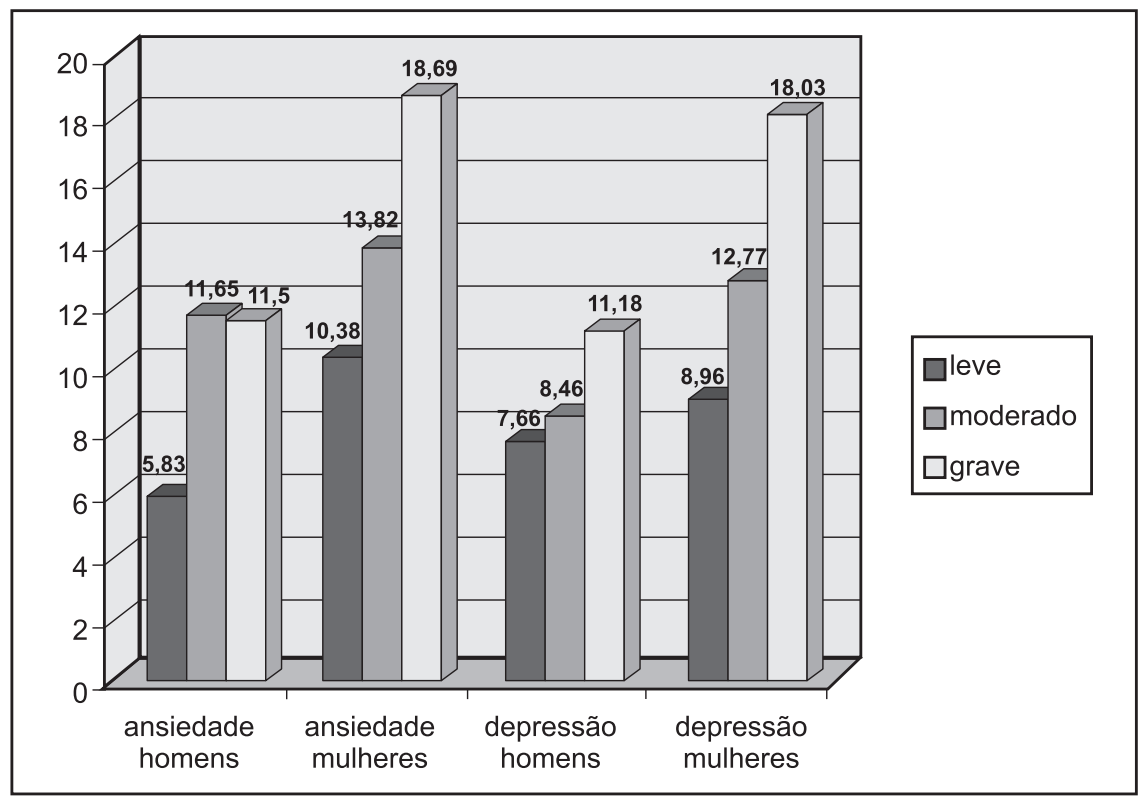

Figura 1 - Sintomas de ansiedade e depressão de acordo com o grau de dependência do tabaco em homens e mulheres 
O mesmo estudo não encontrou associação entre gravidade do tabagismo e depressão entre os sujeitos do sexo masculino, nem entre sintomas de ansiedade e depressão e gravidade do tabagismo entre as mulheres pesquisadas. A pesquisa de Alati et al. ${ }^{26}$ apenas se assemelha ao nosso estudo em seus resultados com relação à falta de associação entre a gravidade da dependência de nicotina e a depressão nos homens.

Esses dados fazem com que se questione o quanto as mulheres deprimidas são mais vulneráveis a se tornarem tabagistas mais graves e o quanto o craving que experimentam pelo tabaco é impulsionado por uma tentativa de aliviar um afeto negativo, o que já havia sido discutido anteriormente por Araújo ${ }^{27}$, e Araújo et al. ${ }^{28,29}$. Assim, poderia se pensar que o fumar estaria muito mais relacionado à capacidade reforçadora da nicotina de alívio do desprazer do que de busca do prazer.

Quando a amostra foi avaliada em sua totalidade incluindo homens e mulheres -, os dependentes de tabaco mais graves apresentaram, porém, tanto um número maior de sintomas de ansiedade quanto de depressão. Fergusson et al. ${ }^{30} \mathrm{e}$ Wilhelm et al. ${ }^{19}$ encontraram esta mesma associação com relação especificamente à depressão, e Castro et al. ${ }^{31}$ obtiveram resultados parecidos com relação aos sintomas de ansiedade. Pode-se pensar que esta associação, no caso dos sintomas de depressão, foi obtida em função dos resultados das mulheres, já que não foi observada esta diferença na amostra do sexo masculino.

$\mathrm{Na}$ avaliação de acordo com o gênero, as mulheres tabagistas apresentaram mais sintomas de ansiedade do que os homens; resultado semelhante ao encontrado por Mykeltun et al. ${ }^{15}$ e Issa et al. ${ }^{20}$. Já no que diz respeito à outra variável pesquisada - os sintomas depressivos -, também foi observado que as mulheres apresentavam sintomas mais intensos do que os homens, o que concorda com o que foi descrito por Husky et al. ${ }^{32}$, Romans et al. ${ }^{33} \mathrm{e}$ Perkins et al. ${ }^{34}$. Issa et al. ${ }^{20}$ destacaram ainda que, após o período de abstinência, os homens têm uma maior facilidade para "superar" o hábito de fumar se comparados com as mulheres, que tendem a descontar a falta do cigarro no excesso de alimentação. Morano ${ }^{35}$ também aponta o fato de as mulheres terem mais recaídas do que os homens e hipotetiza que uma das causas pode ser, além do ganho de peso, a ansiedade, que, em grande parte das vezes, ocorre na síndrome de abstinência da nicotina. Assim, os resultados destes dois estudos podem ser uma das conseqüências da associação entre o gênero e os sintomas não só de ansiedade como de depressão.

Um fator a ser considerado na clínica da dependência química e muito relacionado à ansiedade, à depressão e, conseqüentemente, às recaídas em tabagistas é o craving - que, no Brasil, é chamado de fissura ${ }^{27,28}$. Um achado de Araújo $^{29}$, pesquisando este tema, demonstrou que, entre as mulheres, o craving pelo tabaco estava mais relacionado à capacidade de reforço negativo dessa substância do que a de reforço positivo, isto é, as mulheres desejavam o uso do cigarro para evitar o desprazer e não como forma de aumentar o prazer, levando-nos a inferir que o tabaco pode estar sendo utilizado por esse grupo como automedicação, ou seja, como uma forma de aliviar sentimentos desagradáveis relacionados a sintomas de ansiedade e de depressão.

Percebe-se, pelo que foi discutido, a necessidade de abordagens direcionadas às tabagistas femininas que levem em conta que elas formam um grupo que apresenta mais sintomas de ansiedade e depressão do que os homens e que demanda uma atenção especial para o diagnóstico de possíveis comorbidades em eixo $\mathrm{I}^{7}$, para diminuir as chances de usarem, como já foi salientado, o tabaco como automedicação ${ }^{29}$ e aumentar a chance de manter a abstinência ${ }^{11}$.

Embora os instrumentos utilizados neste estudo não permitam falar em comorbidade por não estabelecer um diagnóstico, eles demonstraram haver associação entre sintomas de ansiedade e depressão e a gravidade do tabagismo. Devemos lembrar que existem hipóteses para tentar explicar comorbidades psiquiátricas em tabagistas: hipóteses causais e não-causais. As hipóteses causais consideram a existência de doenças psiquiátricas como aumento do risco para o tabagismo e responsáveis pelos menores índices de manutenção da abstinência: os pacientes com outro diagnóstico psiquiátrico apresentam de duas a três vezes menos possibilidade de interromper o uso de tabaco do que a população geral. Outra hipótese causal é a de que o tabagismo está implicado no aumento do risco para o aparecimento de outros diagnósticos psiquiátricos. As hipóteses não-causais atribuem essa associação ao ambiente e fatores genéticos compartilhados que predispõem a ambos: dependência de tabaco e outros transtornos psiquiátricos ${ }^{8}$.

Deve-se destacar que as associações encontradas trazem implicações tanto sobre a avaliação como sobre o diagnóstico e tratamento do tabagismo para ambos os sexos: se os profissionais não avaliarem bem os sintomas de ansiedade e de depressão e as comorbidades possivelmente a eles relacionadas, não conseguirão administrar um tratamento adequado para os pacientes, pois o tratamento preconizado para dependentes químicos que tem comorbidades é um tratamento sinérgico, que deve contemplar, simultaneamente, as duas patologias ${ }^{12,36}$.

No momento em que se reconhece a necessidade de uma abordagem conjunta do tabagismo e de suas comorbidades psiquiátricas ${ }^{12,36}$, bem como se tem conhecimento da maior dificuldade nas tentativas de manter a abstinência por parte dos tabagistas mais ansiosos ou depressivos ${ }^{12}$, deve-se defender, junto à comunidade científica, a importância da avaliação dos sintomas de ansiedade e de depressão em homens e mulheres tabagistas.

Entre as limitações observadas estão o fato de os instrumentos utilizados para a avaliação dos sintomas de 
depressão e ansiedade não serem instrumentos diagnósticos e o de poucos sujeitos terem sido classificados com um índice de alto grau de dependência de nicotina, possivelmente em função de os sujeitos serem oriundos da população geral e não de pacientes que buscavam tratamento para tabagismo. A amostragem ter sido realizada de forma não-aleatória (por conveniência) também foi uma limitação deste estudo, que prejudica na generalização de seus resultados.

A cessação do consumo do tabaco é algo bastante complexo e que envolve inúmeras variáveis, sendo assim, quanto mais informações obtivermos a respeito das especificidades dos dependentes dessa substância, mais chances teremos de auxiliá-los na complicada tarefa que é manter a abstinência.

\section{Referências}

1. Achutti A, Menezes AMB. Epidemiologia do tabagismo. In: Achutti A. Guia nacional de prevenção e tratamento do tabagismo. Rio de Janeiro: Vitrô; 2001. p. 9-24.

2. US Department of health and human services: health consequences of smoking cessation: a report of the surgeon general. Washington: US Government Printing Office. 1994;(9):121-258.

3. Carlini EA, Galduróz JCF, Noto AR, Nappo AS. II. Levantamento domiciliar sobre o uso de drogas psicotrópicas no Brasil: estudo envolvendo as 108 maiores cidades do país. São Paulo: UNIFESP; 2005 .

4. Carlini EA, Galduróz JCF, Noto AR, Nappo AS. I. Levantamento domiciliar sobre o uso de drogas psicotrópicas no Brasil: estudo envolvendo as 107 maiores cidades do país. São Paulo: UNIFESP; 2001 .

5. Monteiro CA, Cavalcante TM, Moura EC, Claro RM, Szwarcwald CL. Population-based evidence of a strong decline in the prevalence of smokers in Brazil (1989-2003). Bull World Health Organ. 2007;85:527-34

6. Rodríguez TJ, Hernández AE, Fernández AM. Descripción del consumo de drogas lícitas e ilícitas por género a través de la metodología de pares. Rev Med Chile. 2007;135:449-56.

7. Associação Psiquiátrica Americana. Manual diagnóstico e estatístico de transtornos mentais: texto revisado - DSM-IV-TR ${ }^{\mathrm{TM}}$. $4^{\mathrm{a}}$ ed. Porto Alegre: ArtMed; 2002.

8. Breslau N, Novak SP, Kessler RC. Psychiatric disorders and stages of smoking. Biol Psychiatry. 2004;55(1):69-76.

9. Grant BF, Hasin DS, Chou SP, Stinson FS, Dawson DA. Nicotine dependence and psychiatric disorders in the United States: results from the national epidemiologic survey on alcohol and related conditions. Arch Gen Psychiatry. 2004;61(11):1107-15.

10. Caeiro F, Monteiro O, Aguiar M, Todo-Bom F, Gomes A, Sotto-Mayor $\mathrm{R}$, et al. Four years follow-up in patients seeking medical support for smoking cessation. Rev Port Pneumol. 2007;13(6):37-8.

11. Patkar AA, Vergare MJ, Batra V, Weinstein SP, Leone FT. Tobacco smoking: current concepts in etiology and treatment. Psychiatry. 2003;66(3):183-99.

12. Schmitz N, Kruse J, Kugler J. Disabilities, quality of life, and mental disorders associated with smoking and nicotine dependence. Am J Psychiatry. 2003;160(9):1670-6.

12. Isensee B, Wittchen HU, Stein MB, Hofler M, Lieb R. Smoking increases the risk of panic: findings from a prospective community study. Arch Gen Psychiatry. 2003;60(7):692-700.

13. Lopes FL, Nascimento I, Zin WA, Valença AM, Mezzasalma MA, Figueira I, et al. Smoking and psychiatric disorders: a comorbidity survey. Braz J Med Biol Res. 2002;35(8):961-7.

14. Munaretti CL, Terra MB. Transtornos de ansiedade: um estudo de prevalência e comorbidade com tabagismo em um ambulatório de psiquiatria. J Bras Psiquiatria. 2007;56(2):108-15.

15. Mykletun A, Overland S, Aarø LE, Liabø HM, Stewart R. Smoking in relation to anxiety and depression: evidence from a large population survey: the HUNT study. Eur Psychiatry. 2007; [Epub ahead of print]. 2008;23(2):77-84.

16. Patterson F, Lerman C, Kaufmann V, Neuner G, Audraín-McGovern J. Cigarette smoking practices among american college students: review and future directions. J Am Coll Health. 2004;52(5):203-10.

17. Murphy JM, Horton NJ, Monson RR, Laird NM, Sobol AM, Leighton AH. Cigarette smoking in relation to depression: historical trends the Stirling County Study. Am J Psychiatry. 2003;160(9):1663-9.

18. Lugoboni F, Quaglio G, Pajusco B, Mezzelani P, Lechi A. Association between depressive mood and cigarette smoking in a large Italian sample of smokers intending to quit: implications for treatment. Intern Emerg Med. 2007;2(3):196-201.

19. Wilhelm K, Mitchell P, Slade T, Brownhill S, Andrews G. Prevalence and correlates of DSM-IV major depression in an Australian national survey. J Affect Disord. 2003;75(2):155-62.

20. Issa JS, Perez GH, Diament J, Zavattieri AG, Oliveira KU. Efetividade da Bupropiona no tratamento de pacientes tabagistas com doença cardiovascular. Arq Bras Cardiol. 2007;88:434-40.

21. Fagerström KO. Measuring degree of physical dependence to tobacco smoking with reference to individualization of treatment. Addict Behav. 1978;3(3-4):235-41.

22. Carmo JT, Pueyo A. A adaptação do português do Fagerström test for nicotine dependence (FTND) para avaliar a dependência e tolerância à nicotina em fumantes brasileiros. Rev Bras Med. 2002;59(1/2):73-80.

23. Cunha JA. Manual da versão em português das Escalas Beck. São Paulo: Casa do Psicólogo; 2001.

24. Halty LS, Huttner MD, Oliveira Neto IC, Santos VA, Martins G. Análise da utilização do Questionário de Tolerância de Fagerström (QTF) como instrumento de medida da dependência nicotínica. J Pneumol. 2002;28(4):180-6.

25. Brady K, Grice DE, Dustan L, Randall C. Gender differences in substance use disorders. Am J Psychiatry. 1993;150(11):1707-11.

26. Alati R, Kinner S, Najman JM, Fowler G, Watt K, Green D. Gender differences in the relationships between alcohol, tobacco and mental health in patients attending an emergency department. Alcohol Alcohol. 2004;39(5):463-9.

27. Araujo RB, Oliveira MS, Mansur MA. A validação brasileira do Questionnaire of Smoking Urges. Cad Saude Publica. 2006;22(10):2157-67.

28. Araujo RB, Oliveira MS, Moraes JFD, Pedroso RS, Port F, Castro MGT. Validação da versão brasileira do Questionnaire of Smoking Urges-Brief. Rev Psiq Clín. 2007;34(4);166-75.

29. Araujo RB. O craving em dependentes de tabaco [tese]. Porto Alegre: PUCRS; 2005.

30. Fergusson DM, Goodwin RD, Horwood LJ. Major depression and cigarette smoking: results of a 21-year longitudinal study. Psychol Med. 2003;33(8):1357-67.

31. Castro MG, Oliveira MS, Mores JFD, Miguel AC, Araujo, RB Qualidade de vida e gravidade da dependência de tabaco. Rev Psiquiatr Clin. 2007;34(2):61-7.

32. Husky MM, Mazure CM, Paliwal P, McKee SA. Gender differences in the comorbidity of smoking behavior and major depression. Drug Alcohol Depend. 2008;93(1-2):176-9.

33. Romans SE, Tyas J, Cohen MM, Silverstone T. Gender differences in the symptoms of major depressive disorder. J Nerv Ment Dis. 2007;195(11):905-11.

34. Perkins KA. Sex differences in nicotine versus non-nicotine reinforcement as determinants of tobacco smoking. Exp Clin Psychopharmacol. 1996;(11):199-212.

35. Morano JP. Science of women and addiction. In: Morano JP. A primer on women and tobacco: the leading american epidemic. Reston: American Medical Student Association; 2002. p. 12-20.

36. Zaleski M, Laranjeira RR, Marques ACPR, Ratto L, Romano M, Alves HNP, et al. Diretrizes da Associação Brasileira de Estudos do Álcool e outras Drogas (ABEAD) para o diagnóstico e tratamento de comorbidades psiquiátricas e dependência de álcool e outras substâncias. Rev Bras Psiquiatr. 2006;28(2):142-8. 